\title{
Successful transjugular intrahepatic portal-systemic shunt in an ineligible liver transplant patient with primary biliary cirrhosis with refractory ascites and aplastic anemia
}

\author{
Christopher M. Moore $^{1}$, George Behrens ${ }^{2}$, Hector Ferral ${ }^{2}$, David H. Van Thiel $^{1}$ \\ ${ }^{1}$ Section of Hepatology, Department of Medicine, Rush University Medical Center, Chicago, USA \\ ${ }^{2}$ Section of Interventional Radiology, Department of Radiology, Rush University Medical Center, Chicago, USA \\ Email: david_vanthiel@rush.edu
}

Received 24 October 2012; revised 24 November 2012; accepted 1 December 2012

\begin{abstract}
A transjugular intrahepatic portal-systemic shunt (TIPS) is a standard way to decompress the portal system in cirrhotic patients as a bridge to orthotopic liver transplantation (OLT). Traditionally, TIPS has been indicated for certain portal hypertensive sequelae such as refractory ascites, varices treatment and even hepato-hydrothorax. Herein is a case report on the efficacy of TIPS in an OLT ineligible patient with primary biliary cirrhosis and aplastic anemia who had developed refractive ascites requiring serial paracentesis and esophageal varices. He survived 2.5 years post-TIPS placement and died from complications related to severe leucopenia and the development of sepsis.
\end{abstract}

Keywords: Aplastic Anemia; Ascites; Liver Transplantation; Paracentesis; TIPS

\section{INTRODUCTION}

The Transjugular intrahepatic portal-systemic shunt (TIPS) is a standard interventional radiological technique that is utilized in cirrhotic patients to treat major complications of portal hypertension such as refractory ascites and hepato-hydrothorax, secondary prevention of esophageal varices, and primary prevention of gastric varices [1-4]. TIPS is not a cure for decompensated liver disease, but rather conceived of as a bridge to orthotopic liver transplantation (OLT) [3-5].

OLT is a complex standard-of-care surgery for eligible chronic decompensated liver disease and fulminant liver failure patients [4]. The success of this technology has rested upon improvements in immunosuppressive therapies, surgical technique and appropriate patient selection. The MELD score [1-3], albeit imperfect, has been instrumental in risk stratifying patients by overall 90 day mortality. The score is the product of a mathematical equation comprising serum bilirubin, creatinine and INR that is updated periodically on transplant eligible patients.

In brief, the TIPS procedure bypasses the hepatic parenchyma by means of a metallic stent directly linking a branch of the portal vein to a hepatic vein with resultant decompres sion of the portal system and high blood flow into the cardiopulmonary system [3,4]. Given the technical challenges of TIPS, and the expected physiologic changes induced, eligible patients must fulfill a number of criteria including absence of sepsis, absence of severe cardiopulmonary disease, and few prior episodes of hepatic encephalopathy (HE). More comprehensively, it has been determined that the best candidates for TIPS are usually those patients with a Childs-Turcotte-Pugh (CTP) class of A, or a model for end-stage liver disease (MELD) score of $<14$ [1-3]. TIPS has known side-effects including bleeding, infection, stent malposition/thrombosis, HE, abdominal pain and hepatic injury/necrosis.

Herein is a report of a case for the successful utilization of a TIPS procedure in a patient with decompensated primary biliary cirrhosis (PBC), deemed ineligible for OLT given the high morbidity and mortality of his aplastic anemia.

\section{CASE REPORT}

A 62-year-old white male with underlying aplastic anemia and PBC developed portal hypertensive sequelae including diuretic-resistant refractory ascites and esophageal varices. His diagnosis of aplastic anemia was made in 2007. He had been treated previously for the aplastic anemia with sequential therapies consisting of steroids, rituximab, and cyclosporine. He was receiving intravenous immunoglobulin therapy at the time of his referral. His diagnosis of PBC was based upon a combination of data, including: an elevated serum alkaline phosphatase, anti-mitochondrial antibody positivity, increased total serum cholesterol and IgM levels, and a computed to- 
mography (CT) scan documented hepatosplenomegaly with increased hepatic heterogenicity.

Approximately 1 year prior to our initial evaluation, he developed portal hypertensive ascites which was initially responsive to sodium diet restriction and diuretic therapy, consisting of a combination of furosemide and spironolactone at $120 \mathrm{mg}$ and $300 \mathrm{mg}$ per day, respectively. However, his blood urea nitrogen (BUN) and serum creatinine levels increased over time, reflecting worsening kidney injury, such that he became diuretic-resistant. He consequently required serial large-volume paracentesis (LVP) every two weeks for a three-month duration in order to manage the rapidly re-accumulating ascitic fluid in the setting of inadequate diuresis. Additionally, he underwent standard esophagogastric-duodenoscopy (EGD) for varices surveillance, and was found to have large esophageal varices which were band ligated. Subsequently, he was maintained on beta-blocker therapy for variceal prophylaxis. He has never had a variceal bleeding episode. He has never had an episode of HE. Given his known diagnosis of aplastic anemia, the referring heaptologists were concerned about the risks of future esophageal variceal bleeding and consequence of continued LVP upon his physiologic reserve capacity and state of nutrition. Thus, he was referred for more definitive treatment in the form of OLT. Additionally, it was noted that he was quite malnourished with severe generalized muscle wasting and a markedly protuberant abdomen with large-volume ascites. His overall medical history was reviewed thoroughly by the multi-disciplinary liver transplant team, which declined him for OLT because of his aplastic anemia and a related life expectancy of less than 5 years even with an OLT.

Following this decision, he was evaluated for a TIPS procedure recognizing that it would not be as a bridge therapy to OLT, but rather, that it would simplify his continued medical management and reduce the catabolic state in the setting of portal hypertension and aplastic anemia. He underwent a diagnostic and therapeutic LVP, with removal of $2.1 \mathrm{~L}$ of culture negative ascitic fluid, containing 10 white blood cells (WBC) per $\mathrm{mm}^{3}$, and an ascitic albumin level of $2.0 \mathrm{~g} / \mathrm{d}$, which when subtracted from his serum albumin level yielded a serum ascitesalbumin gradient (SAAG) of 1.2, consistent with his diagnosis of portal hypertension. A triple-phase CT scan re-demonstrated findings consistent with advanced cirrhosis with arterial-venous shunts, a few hepatic cysts, no focal lesions, and patent hepatic, portal and splenic veins, as well as large esophageal varices.

Based upon these findings and further evaluation yielding no significant cardiopulmonary disease, a TIPS procedure was offered to the patient, who at the time had the following laboratory values, listed in Table 1. The TIPS procedure was performed in standard fashion with the implementation of $28 \mathrm{~cm} \times 10 \mathrm{~mm}$ Viatorr ${ }^{\odot}$ polytetrafluoroethylene (PTFE)-covered stent without any complications. Pre- and post-TIPS hepatic pressures as well as the hepatic wedge pressure gradient are documented in Table 2 and demonstrate adequate portal venous decompression. The patient did well post-TIPS procedure and was discharged home the following day.

Table 1. Clinical laboratory findings in the patient ${ }^{\dagger}$.

\begin{tabular}{ccccc}
\hline & Pre-TIPS & Day of TIPS & Day after TIPS & Normal value \\
\hline WBC count & $\mathbf{0 . 8 1}$ & $\mathbf{1 . 2 7}$ & $\mathbf{1 . 4 5}$ & $4-10 \times 10^{3} / \mu \mathrm{l}$ \\
Hemoglobin & $\mathbf{7 . 6}$ & $\mathbf{9 . 7}$ & $\mathbf{1 0 . 7}$ & $13.5-17.5 \mathrm{~g} / \mathrm{dl}$ \\
MCV & 94.3 & 90.8 & 90.1 & $82-103 \mathrm{fl}$ \\
Platelet count & $\mathbf{4 2}$ & $\mathbf{3 5}$ & $\mathbf{2 6}$ & $150-399 \times 10^{3} / \mu \mathrm{l}$ \\
BUN & $\mathbf{3 4}$ & $\mathbf{3 0}$ & $\mathbf{2 9}$ & $8-21 \mathrm{mg} / \mathrm{dl}$ \\
Creatinine & 1.1 & 1.1 & 1.1 & $0.6-1.3 \mathrm{mg} / \mathrm{dl}$ \\
Blood glucose & 94 & $\mathbf{1 3 5}$ & 93 & $60-109 \mathrm{mg} / \mathrm{dl}$ \\
Total protein & $\mathbf{3 0}$ & $\mathbf{5 . 6}$ & $\mathbf{5 . 5}-8.2 \mathrm{~g} / \mathrm{dl}$ \\
Albumin & $\mathbf{2 . 5}$ & 1.3 & $\mathbf{2 . 5}$ & $3.5-5.0 \mathrm{~g} / \mathrm{dl}$ \\
Total bilirubin & 1.3 & 35 & $\mathbf{1 . 4}$ & $0.2-1.3 \mathrm{mg} / \mathrm{d}$ \\
AST & 20 & 20 & 36 & $3-44 \mathrm{U} / \mathrm{l}$ \\
ALT & 13 & $X$ & 24 & $0-40 \mathrm{U} / \mathrm{l}$ \\
Prothrombin time & 13.7 & $\mathrm{X}$ & $\mathbf{1 5 . 7}$ & $9.5-14.0 \mathrm{~s}$ \\
INR & 1.17 & $\mathbf{X}$ & $\mathbf{1 . 3 9}$ & $0.83-1.23$ (no unit) \\
aPTT & $\mathbf{3 4}$ & $\mathrm{X}$ & 23 - 33 s \\
\hline
\end{tabular}

${ }^{\dagger} \mathrm{WBC}=$ white blood cells; $\mathrm{MCV}=$ mean corpuscular volume; $\mathrm{BUN}=$ blood urea nitrogen; $\mathrm{AST}=$ aspartate aminotransferase; ALT = alanine aminotransferase; INR= international normalized ratio; aPTT = activated partial thromboplastin time; X = not available. Abnormal data in bold font. 
Table 2. Calculated portal vein pressures (mmHg).

\begin{tabular}{cccc}
\hline & Pre-TIPS & Post-TIPS & Normal value \\
\hline Hepatic vein & 19 & 20 & $<5$ \\
Wedged portal vein & 32 & 27 & $5-10$ \\
Gradient & 13 & 7 & $0-5$ \\
\hline
\end{tabular}

At a follow-up outpatient visit two weeks later, an abdominal ultrasound (US) with a complete duplex Doppler study was performed and documented peak systolic velocities at the portal vein end of the TIPS, mid TIPS, and hepatic vein end of the TIPS of 57, 163 and 119 $\mathrm{cm} / \mathrm{sec}$ with no high velocity jets, respectively. In the following weeks, the patient's ascites resolved, and his diuretic requirements were reduced to $20 \mathrm{mg}$ furosemide and $50 \mathrm{mg}$ spironolactone per day in the setting of stable kidney disease and an adequate diuresis. In the post-TIPS state, he no longer required paracentesis, his esophageal varices were decompressed, his nutritional state and overall clinical appearance improved. Most importantly, he did not develop any complications of his TIPS procedure and survived an additional 2.5 years at which time he died as a result of severe leucopenic sepsis.

\section{DISCUSSION}

Cirrhosis is manifested by an extensive fibronodular replacement of the liver parenchyma with resultant portal hypertension and progressive synthetic dysfunction [4]. The development of portal hypertension leads to a number of complications including HE, ascites and variceal bleeding, which are each associated with an increased morbidity and mortality rate [2-5]. Ascites is treated with a combination of dietary sodium restriction and diuretics, primarily consisting of furosemide and spironolactone. In approximately $10 \%$ of ascites patients, a diagnosis of refractory ascites develops wherein they no longer respond to or cannot tolerate these diuretic therapies. In such cases, LVP is instituted, and while efficacious, is associated with complications consisting of peritoneal bleeding, infection, and increased catabolic rate. Furthermore, there are ongoing concerns of long-term cost and quality-of-life issues [2,3,6].

It is within this setting that the theory and implementation of TIPS arose. TIPS provides a non-surgical method of delivering blood from the portal to hepatic venous system through the utilization of a transhepatic bypass stent [3]. It is generally accepted that a TIPS procedure is utilized best in patients having been classified as CTP class A patient or with a MELD score $<14$ who is awaiting OLT, and who has experienced a number of portal hypertensive complications consisting of refractory ascites or variceal bleeding. Other indications for a TIPS include the development of a hepato-hydrothorax, al- though experience with this problem is less-well documented [3-5]. Furthermore, given the expected physiologic effect of TIPS a number of contra-indications to its implementation have been identified such as congestive heart failure and severe pulmonary hypertension. While TIPS has been successful in ameliorating the complications of portal hypertension, its long-term effectiveness, effect on quality-of-life, and health-care cost have also been matters of debate when compared to serial LVP [2,3, 6]. In part, some of these issues revolve around the studies of uncovered TIPS stent compared to LVP, whereas in the current period covered TIPS stents, which are less likely to become occluded, are the standard-of-care [7].

In this case report, the patient had several common portal hypertensive sequelae of his PBC, including esophageal varices and refractory ascites. Furthermore, the ascites required serial LVP, which despite being a fairly common procedure, is nevertheless associated with complications and costs that are substantial over time. Worse still is the patient's underlying aplastic anemia, a disease process itself manifested by pancytopenia including symptomatic anemia, hemorrhage, and a risk of infection. In addition, there is also an increased risk for the development of hematologic cancer [8]. It was this underlying disease process and its associated morbidity and mortality that both excluded the patient from OLT consideration and made continued LVP a prohibitive option given the well-recognized probability of complications over time.

Given these facts, the present case report is interesting for several reasons. Firstly, it is as best as we can determine the only case in which a TIPS procedure has been performed in a cirrhotic individual with underlying aplastic anemia. Importantly, the TIPS procedure was successful in reducing his ascites and the need for repetitive LVP as well as decompressing his esophageal varices. Importantly, no complications were experienced in the procedure or experienced in his post-procedural followup. Moreover, he was able to reduce his diuretic requirements and reduce his catabolic state. The benefits to his esophageal varices were important, as any future bleeding episodes would be complicated not only by his baseline anemia and thrombocytopenia, but also in the difficulty of finding an appropriate blood match given the number of anti-platelet antibodies he had developed over the preceding years of transfusion.

As he no longer required LVP, his nutrition status, as assessed by physical appearance improved. LVP results in significant ascitic fluid protein losses when compared to diuretic therapy [9]. Additionally, in the post-TIPS setting in the absence of ascites there is an associated increased mobility, energy and protein intake compared to continued LVP [10]. Post-TIPS, his quality-of-life improved, a general finding well-documented in the litera- 
ture [6], although this metric was not quantitatively studied in the patient reported. The underlying aplastic anemia did not precipitate any perceived complications regarding the TIPS procedure per se or his clinical status afterwards until his death as a result of leucopenic sepsis 2.5 years later. These outcomes support the pragmatism of the therapeutic goals: that he was appropriately denied an OLT given his underlying high-mortality state, but that he could benefit clinically from a non-standard indication TIPS procedure given the severity of his portal hypertension and aplastic anemia. Organ allocation is especially stringent for OLT given the relative paucity of available donors to recipients.

In summary, this case report details the non-standard application of a TIPS procedure in a PBC OLT ineligible patient with aplastic anemia and refractory ascites requiring serial LVP. The TIPS procedure was effective in ameliorating sequelae of his portal hypertension and was accomplished without any complications related to his underlying aplastic anemia. Careful application of such a procedure, although not traditionally indicated for such a patient as in this case report, can have reasonable benefits upon quality of life, and possibly even health-care cost.

\section{REFERENCES}

[1] Rössle, M., Ochs, A., Gulberg, V., Siegerstetter, V., Holl, J., Deibert, P., et al. (2000) A comparison of paracentesis and transjugular intrahepatic portosystemic shunting in patients with ascites. New England Journal of Medicine, 342, 1701-1707. doi:10.1056/NEJM200006083422303

[2] Saab, S., Nieto, J.M., Lewis, S.K. and Runyon, B.A. (2006) TIPS versus paracentesis for cirrhotic patients with refractory ascites. Cochrane Database of Systematic
Reviews, 18, Article ID: CD004889.

[3] Boyer, T.D. and Haskal, Z.J. (2010) American association for the study of liver diseases. The role of transjugular intrahepatic portosystemic shunt (TIPS) in the management of portal hypertension: Update 2009. Hepatology, 51, 306. doi:10.1002/hep.23383

[4] Lefton, H.B., Rosa, A. and Cohen, M. (2009) Diagnosis and epidemiology of cirrhosis. Medical Clinics of North America, 93, 787-799. doi:10.1016/j.mcna.2009.03.002

[5] Runyon, B.A. (2009) AASLD practice guidelines committee. Management of adult patients with ascites due to cirrhosis: An update. Hepatology, 49, 2087-2107. doi:10.1002/hep.22853

[6] Campbell, M.S., Brensinger, C.M., Sanyal, A.J., et al., Quality of life in refractory ascites: Transjugular intrahepatic portal-systemic shunting versus medical therapy. Hepatology, 42, 635-640. doi:10.1002/hep.20840

[7] Fidelman, N., Kwan, S.W., Laberge, J.M., Gordon, R.L., Ring, E.J. and Kerlan Jr., R.K. (2012) The transjugular intrahepatic portosystemic shunt: An update. American Journal of Roentgenology, 199, 746-755. doi:10.2214/AJR.12.9101

[8] Young, N.S., Bacigalupo, A. and Marsh, J.C. (2010) Aplastic anemia: Pathophysiology and treatment. Biology of Blood and Marrow Transplantation, 16, S119-S125. doi:10.1016/j.bbmt.2009.09.013

[9] Runyon, B.A., Antillon, M.R. and McHutchison, J.G. (1992) Diuresis increases ascitic fluid opsonic activity in patients who survive spontaneous bacterial peritonitis. Journal of Hepatology, 14, 249-252. doi:10.1016/0168-8278(92)90166-M

[10] Plauth, M., Schutz, T., Buckendahl, D.P., et al. (2004) Weight gain after transjugular intrahepatic portosystemic shunt is associated with improvement in body composition in malnourished patients with cirrhosis and hypermetabolism. Journal of Hepatology, 40, 228-233. doi:10.1016/j.jhep.2003.10.011 